\title{
The Frequency Stability of Airflow-induced Acoustic Vibration Piezoelectric Generator
}

\author{
Jian Zhang ${ }^{1}$, Jiacun $\mathrm{Sun}^{2}$, Jianyu Cai ${ }^{2}$, Hejuan $\mathrm{Chen}^{2}$ and Chi $\mathrm{Yao}^{3}$ \\ ${ }^{1}$ Taizhou Institute ,Nanjing University of Science\&Technology, Taizhou 225300,Jiangsu,China \\ ${ }^{2}$ School of Mechanical Engineering ,Nanjing University of Science\&Technology,Nanjing 210094,Jiangsu,China \\ ${ }^{3}$ Northwest Industry Group Corporation, Xi'an 710000 ,Shanxi, ,China
}

\begin{abstract}
The Wind Turbines is excited by natural wind in working environment. The piezoelectric generator gets concern in self-driven, self-powered, micro-electromechanical systems for its environmentally friendly feature, and easiness to get miniaturization. Power Quality is one of the necessary conditions for the normal operation of the generator system, while the output voltage and frequency are the main indicators to measure electrical energy. At rated voltage and power, stable frequency is the guarantee for system working accuracy. According to frequency pump principle, it is effective for piezoelectric transducer to increase gathering power through the adjustment of oscillator parameter and amplification of vibration frequency. The designed Airflow-induced Acoustic -vibration Piezoelectric Generator makes the frequency get close to the natural frequency of the piezoelectric transducer. The result shows that: the frequency of the sound pressure is stable, the vibration source frequency is amplified.
\end{abstract}

Keywords-component; formatting; style; styling; insert

\section{INTRODUCTION}

The Wind Turbines is excited by natural wind in working environment, the wind speed in the range of $1 \sim 10 \mathrm{~m} / \mathrm{s}$. The horizontal or vertical axis turbine rotary incentive structure is adopted currently, and the turbine blades is pushed by wind or airflow directly to drive the magnetic transducer to generate power, at the same time, the generator is huge. Generally, as to common self-powered system equipment, such a huge generator is suitable for the circumstances that lack apt place to install with complex structure, high cost, loud noise, and electromagnetic pollution, etc.. However, the piezoelectric generator gets concern in self-driven, self-powered, microelectromechanical systems for its environmentally friendly feature, and easiness to get miniaturization.

Power Quality is one of the necessary conditions for the normal operation of the generator system, while the output voltage and frequency are the main indicators to measure electrical energy. At rated voltage and power, stable frequency is the guarantee for system working accuracy. The piezoelectric vibration generator is single-phase $\mathrm{AC}$ generator, which was capacitive, whose core component are the exciting mechanism and piezoelectric transducers, the vibration source is excited by airflow, the piezoelectric transducer is an energy harvesting device by forced vibration, and the maximum convert power can be get when the frequency is equal to the natural frequency of the system. However, as to the piezoelectric energy harvesters for persistent base motions at the mode of linear vibration, the energy conversion efficiency is low at optimum impedance matching, which is not exceeding $25 \%$, and the output power is still low. The vibration energy harvesting induced by natural wind in working environment or air stream generated by oncoming air, whose core component is motivate institution and forced vibration piezoelectric transducer. The fixed frequency of the common cantilever piezoelectric transducer structure is only a few dozenS to a few hundred hertz, and the impulse of aerodynamic force is irregular at rather low frequency. The actual systems are often in a mismatch state due to the structure, materials, technology and other errors, so there would be no resonance, the impedance mismatch and system frequency instability also influence seriously the output power of the generator.

According to frequency pump principle, it is effective for piezoelectric transducer to increase gathering power through the adjustment of oscillator parameter and amplification of vibration frequency. The designed Airflow-induced Acoustic vibration Piezoelectric Generator makes the frequency get close to the natural frequency of the piezoelectric transducer. This paper has done output performance test to analyze the relations between Single-phase AC voltage output, output power, frequency, input stimulus airflow, and the load.

\section{WORKING PRINCIPLE OF AIRFLOW-INDUCED ACOUSTIC - VIBRATION PIEZOELECTRIC GENERATOR}

Outcome inflow works on object at some flow oscillation frequency, independent of the natural frequency and vibration modes, oscillating force generated the object is irrelevant, which is a fluid resonance phenomenon of forced vibration. External energy in the laser usually inputs in the form of light or current ted to the lasing medium, exciting the electron into a higher energy level, which is a laser "pumping" phenomenon. According to frequency pump principle, by producing more vibrations within the same time, and increasing oscillator frequency before the input excitation gets piezoelectric transducer, the output power of the piezoelectric generator can be greatly increased. Figure 1 is the schematic diagram of airflow-induced acoustic -vibration piezoelectric generator based on excitation and frequency pumping design principle, which is made up of airflow modulation control system and piezoelectric vibration energy generation systems, gas caused by acoustic vibrations of the piezoelectric generator ${ }^{[1-4]}$ 
schematic. It consists of two parts airflow modulation control system and the vibration energy of the piezoelectric power generation system. The piezoelectric generator output voltage $V_{0}(t)$ and the power $P_{0}(t)$ depending on the bottom of the cavity resonance sound pressure $P_{\mathrm{i}}(t)$, the sound wave $P_{1}(t)$ is a spout at the "Karman vortex street" Karman Vortex Street excited, and this vortex is the inflow $V_{\mathrm{i}}(t)$ induced. $P_{\mathrm{i}}(t)$ and $P_{1}(t)$ is the fluctuating pressure, their frequency and close, in fact, this is a frequency capture process. Based on the experiment, the test $P_{\mathrm{i}}(t), V_{0}(t)$ of the frequency, to obtain the frequency characteristic.

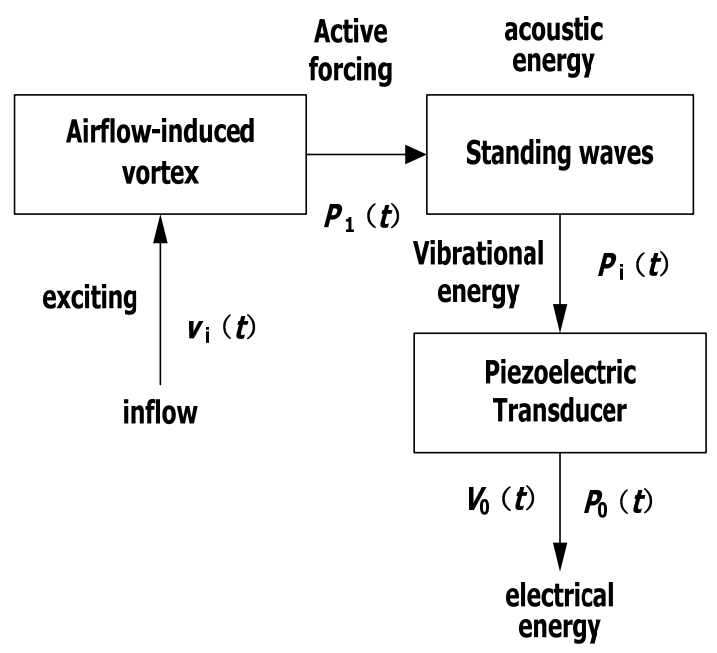

FIGURE I. AIRFLOW-INDUCED ACOUSTIC-VIBRATION PIEZOELECTRIC GENERATOR

\section{THE MEASUREMENT PRINCIPLE OF DIRECT VOLTAGE WAVEFORM OF EXCITATION FORCE FREQUENCY}

Figure I Equivalent equations of piezoelectric transducer

$$
m \ddot{u}+c \dot{u}+k_{E} u=F_{e x}-F_{p}
$$

Where, $\mathrm{m}$ is equivalent quality of the piezoelectric transducer; $\mathrm{u}$ is equivalent shift; $k_{E}$ is equivalent stiffness; $\mathrm{c}$ is equivalent damping coefficient; $F_{e x}$ is excitation force, $F_{p}$ is piezoelectric force, as in

$$
\left\{\begin{array}{c}
F_{\mathrm{p}}=\left(k_{\mathrm{PD}}-\frac{\alpha^{2}}{C_{\mathrm{p}}}\right) u+\frac{\alpha A}{b C_{\mathrm{p}}} V \\
V=\frac{\alpha b}{A} u-\frac{b^{2} C_{\mathrm{p}}}{A^{2}} \int_{0}^{t} I \mathrm{~d} t
\end{array}\right.
$$

A, b are separately surface area and thickness of piezoelectric patches, V and I are separately output voltage and current of piezoelectric patches, $k_{\mathrm{PD}}$ is equivalent stiffness of piezoelectric patches under open electric circuit, $k_{\mathrm{PD}}=k_{\alpha X}{ }^{D} A / b, k_{2 \pi}{ }^{D}$ is stiffness constant of open circuit; $C_{\mathrm{p}}$ is stable clamp capacitance of piezoelectric patches; $c_{\mathrm{p}}=\beta_{33}{ }^{S} A / b, \beta_{33}{ }^{s}$ is clamp dielectric isolation, $\alpha$ is piezoelectric stress factor, $\alpha=k_{33} A / b$.

According to the theory of forced vibration, general solution of formula (1) is transient vibration decreased as the index, which is meaningful only in a period of time after the vibration started, so the general solution of formula(1) is ignored, which only takes the special solution $u$ into consideration, exciting force $F_{\mathrm{ex}}=F_{0} \sin \omega \mathrm{t}, \omega=2 \pi f$,f is vibration frequency. $u$ is Sine function, whose frequency is $f$, the amplitude of output voltage, $\mathrm{V}$ and current, I are related to $F_{0}$, and irrelevant to energy harvesting circuit. According to formula(2), frequency of $\mathrm{V}$ is equal to $f$, the frequency of $u$. Thus, exciting force frequency can be accessed directly by measuring voltage frequency, which is the measurement principle of direct voltage waveform of excitation force.

\section{PRESSURE OF THE RESONATOR BOTTOM, TESTING EXPERIMENT OF THE GENERATOR OUTPUT VOLTAGE}

\section{A. The Natural Frequency of Piezoelectric Vibrator and Transducer}

Figure 2 is experiment system, which consists of gas source, testing instruments and experimental products, etc. Minimum impedance value and fixed frequency values of piezoelectric oscillator in free state and fixed piezoelectric transducer are separately measured by impedance analyzer. Table 1 is natural frequency and impedance values of piezoelectric oscillator and piezoelectric transducer of generator $1 \# \sim 5 \#$, and the fixed frequency values are separately $(\mathrm{kHz}): 5.25,5.65,7.20,5.00,5.25$. Seen from the values in Table I the natural frequency of the piezoelectric transducer is nearly half of that of piezoelectric vibrator, while the impedance value of the piezoelectric transducer is over twice than that of piezoelectric vibrator.

TABLE I. MEASUREMENT VALUE OF THE MINIMUM IMPEDANCE VALUE AND FIXED FREQUENCY

\begin{tabular}{c|c|c|c|c}
\hline \multirow{2}{*}{ NO. } & \multicolumn{2}{|c|}{ piezoelectric vibrator } & \multicolumn{2}{c}{$\begin{array}{c}\text { piezoelectric } \\
\text { transducer }\end{array}$} \\
\cline { 2 - 5 } & $\begin{array}{c}\text { frequency } \\
/ \mathbf{k H z}\end{array}$ & $\begin{array}{c}\text { Impedance } \\
/ \mathbf{k} \boldsymbol{\Omega}\end{array}$ & $\begin{array}{c}\text { natural } \\
\text { frequency } \\
/ \mathbf{k H z}\end{array}$ & $\begin{array}{c}\text { Equivalent } \\
\text { impedance } \\
/ \mathbf{k} \boldsymbol{\Omega}\end{array}$ \\
\hline $1 \#$ & 12.50 & 2.00 & 5.25 & 4.70 \\
\hline $2 \#$ & 13.50 & 1.20 & 5.65 & 4.00 \\
\hline $3 \#$ & 13.50 & 1.90 & 7.20 & 3.72 \\
\hline $4 \#$ & 14.50 & 2.17 & 5.00 & 6.70 \\
\hline $5 \#$ & 12.00 & 2.10 & 5.25 & 5.75 \\
\hline
\end{tabular}



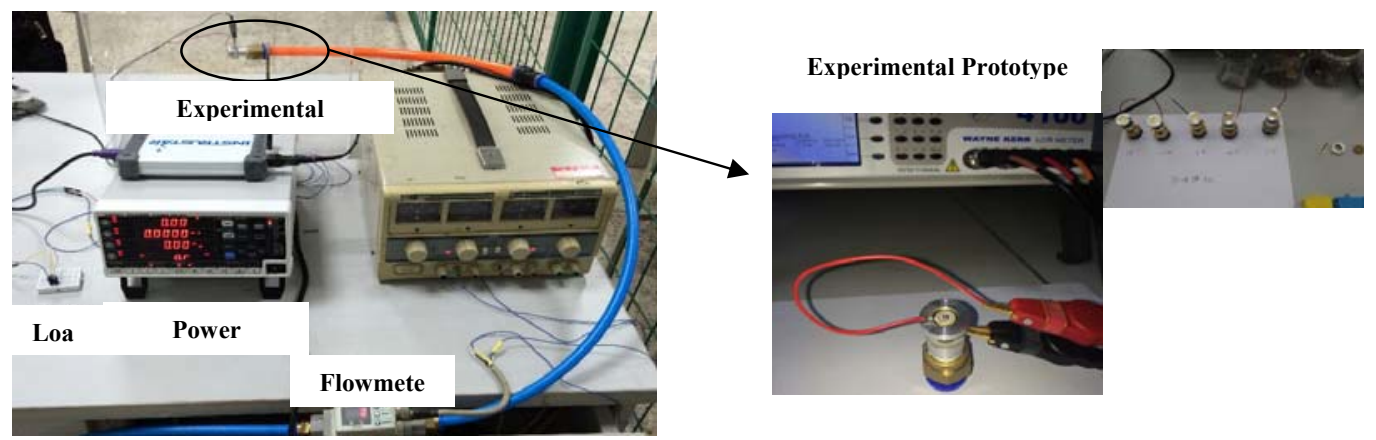

FIGURE II. FLOW CHART OF THE EXPERIMENT

\section{B. Testing curve of vi- $f P i$}

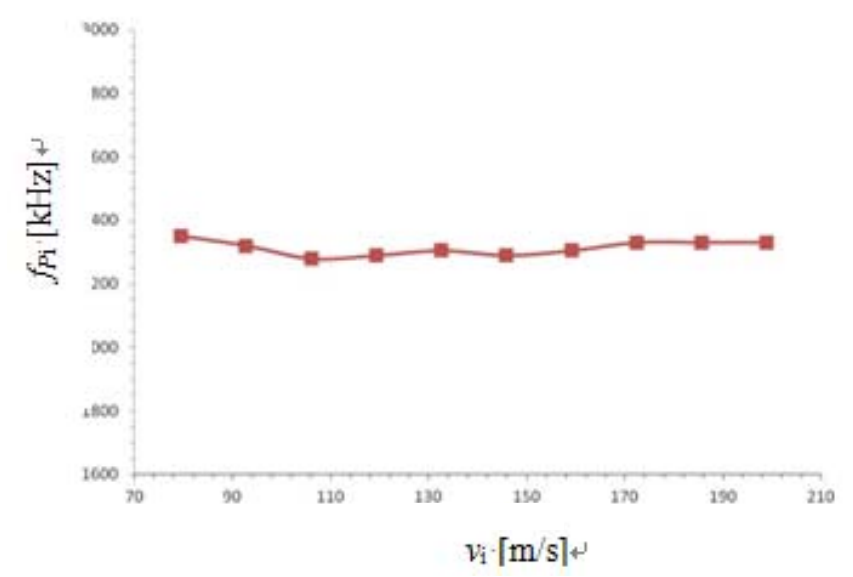

FIGURE III. VI-FPI CURVE

Figure 3 is $v_{\mathrm{i}}-f_{P_{\mathrm{i}}}$ testing curve of $1 \#, f_{P \mathrm{i}}$ in the figure is obtained through FFT shift of resonant cavity (Pressure) curve. $f_{P \mathrm{i}}$ is $2.25 \sim 2.35 \mathrm{kHz}, v_{\mathrm{i}}$ is a voltage at $2.3 \pm 0.05 \mathrm{kHz}$. The frequency error is about $50 \mathrm{~Hz}$.

C. Testing curve of $v i-f$

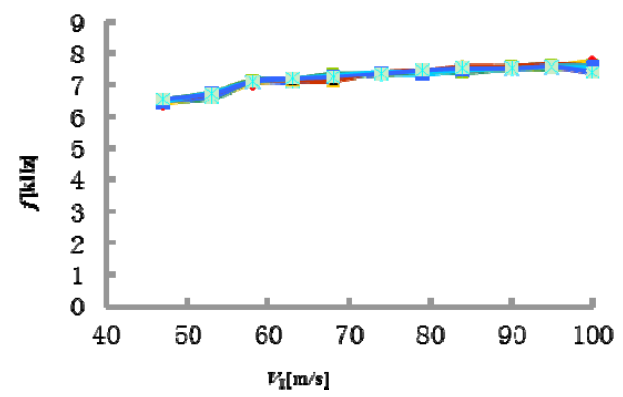

FIGURE IV. Vi- F Curve

Figure 4 is $v_{i}-f$ testing curve of $1 \#, f$ is obtained through FFT shift of resonant cavity pressure) curve. According to the datas in the figure, $f_{>} f_{P \mathrm{i}}$, showing frequency is enlarged. Besides, $f$ fluctuates between $6.0 \sim 7.5 \mathrm{kHz}$, which is
$6.75 \pm 0.75 \mathrm{kHz}$ with $v_{\mathrm{i}}$ varies, frequency error is about $0.75 \mathrm{kHz}$. Figure 5 is output voltage $V_{0}(t)$ curve of generator $1 \#$, showing its variation regulation, frequency value reaches $6.0 \mathrm{kHz}$. Figure 6, output voltage and FFT shift curve of generator 1\#.

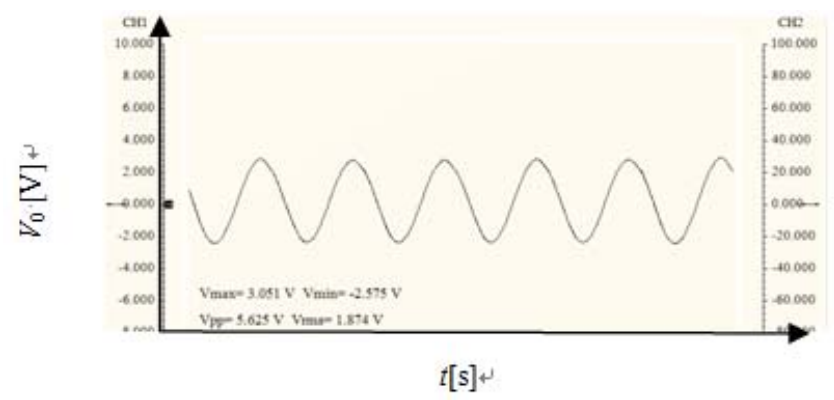

FIGURE V. 1\# V0 (T) CURVE

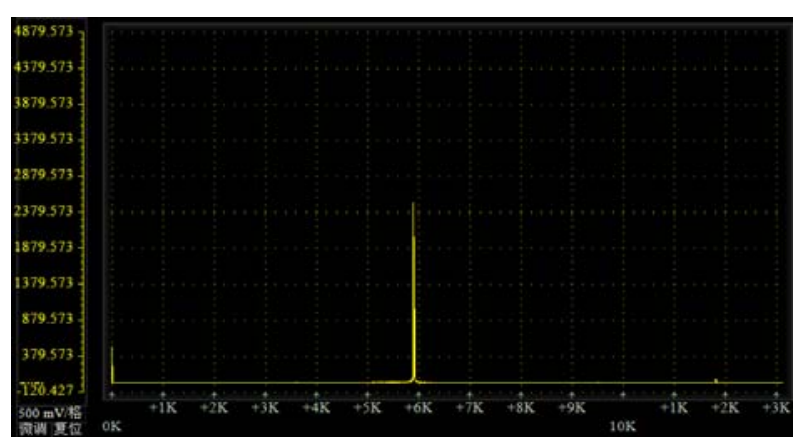

FIGURE VI. 1\# FFT CURVE

\section{CONCLUSION}

(1) The frequency of the sound pressure is stable, which does not vary with the velocity of the inflow.

(2) The generator outputs single-phase AC voltage, the amplitude and frequency of which remains stable, not vary with the velocity of the inflow.

(3) The frequency of the generator's output single-phase $\mathrm{AC}$ voltage is larger than the frequency of the sound pressure, which proved the vibration source frequency is amplified. 


\section{ACKNOWLEDGEMENT}

This research is financially supported by the National Natural Science Foundation of China under contract No. 51377084 .

\section{REFERENCES}

[1] Peng He, Simulation of flow field in the airflow control inlet of the new pneumatic piezoelectric generator of missile-borne fuze.[D].Nanjing: Nanjing University of Science\&Technology,2012.

[2] $\mathrm{Na} \mathrm{Lv}$, Numerical simulation of turbulent flow in the airflow control inlet of the new pneumatic piezoelectric generator of missile-borne fuze.[D].Nanjing: Nanjing University of Science\&Technology,2012.

[3] Huajie Zou, Hejuan Chen,Yi Liang,Qi Jiang,Bin Liu,Junhong Wang, Study of drive characterization of fuze vibration piezoelectric generator based on airflow-induced sound.2015.

[4] Xiaoguang Zhu, Technologies for the airflow induced vibration and experimentation on the miniature vibration piezoelectric generator. [D].Nanjing: Nanjing University of Science\&Technology,2012.

[5] S Priya,DJ Inman.Energy Harvesting Technologies. Springer Science\&Business Media . 2009

[6] Wei Qu, Energy harvesting technology for the fuze airflow induced vibration piezoelectric generator.2014. 